\title{
Gradient representations in ReLU networks as similarity functions
}

\author{
Dániel Rácz ${ }^{1,3}$ and Bálint Daróczy ${ }^{2,3}$ \\ 1 - Eötvos Loránd University, Institute of Mathematics \\ Pázmány Péter sétány 1/C, H-1117, Budapest, Hungary \\ 2 - Université catholique de Louvain, INMA/ICTEAM \\ Avenue Georges Lemaître 4, B-1348, Louvain-la-Neuve, Belgium \\ 3 - Institute for Computer Science and Control, SZTAKI/ELKH \\ Kende utca 13-17, H-1111, Budapest, Hungary \\ e-mail: racz.daniel@sztaki.hu, balint.daroczy@uclouvain.be
}

\begin{abstract}
Feed-forward networks can be interpreted as mappings with linear decision surfaces at the level of the last layer. We investigate how the tangent space of the network can be exploited to refine the decision in case of ReLU (Rectified Linear Unit) activations. We show that a simple Riemannian metric parametrized on the parameters of the network forms a similarity function at least as good as the original network and we suggest a sparse metric to increase the similarity gap.
\end{abstract}

\section{Introduction}

We consider feed-forward neural networks with ReLU activations to examine how the network's final representation space connected to the gradient structure of the network. Our motivation is twofold: recently discovered knowledge about ReLU networks $[1,2,3]$ and recent results about higher order optimization methods [4]. In a way, many of the existing machine learning problems can be investigated as statistical learning problems, therefore information geometry [5] plays an important role. It was shown in [6] that over the parameter space of a neural network we can often determine a Riemannian manifold based on an error or loss function, moreover the tangent bundle on specific Riemannian metrics, e.g. Fisher information, has unique invariance properties [7].

Our main hypothesis is that in feed-forward ReLU networks we can utilize the relation of the parameters and the output due the homogeneity property of the activation functions. Therefore, we investigate the gradient structure of the network output w.r.t. the parameters and exploit the space induced by the partial derivatives together with a metric as a representation of data points. Albeit the inner product space of the tangent bundle is quadratic, there are welldefined underlying structures in the tangent space specific to ReLU networks. In this paper we introduce several similarity functions [8] based on a blockdiagonal, sparse metric and we inspect how they relate to the similarity induced

${ }^{*}$ The research was supported by the Hungarian Ministry of Innovation and Technology NRDI Office within the framework of the Artificial Intelligence National Laboratory Program. BD was supported by MIS "Learning from Pairwise Comparisons" of the F.R.S.-FNRS and by MTA Premium Postdoctoral Grant 2018. 
by the network itself by measuring the similarity gap, the difference in expected similarity between point pairs with the same label and point pairs with different labels.

\subsection{Related work}

The geometrical properties of the underlying loss manifold of neural networks was used as a general framework for optimization in classification and generative models [6]. Furthermore, Martens and Grosse [4] approximated Amari's natural gradient [5] for feed-forward neural networks with block partitioning the Fisher information matrix by exploiting the structure of the network. The partial gradients were used as representations in classification given a known generative probability density function, e.g. through an approximated Fisher kernel, in [9] where the authors approximated the metric with the diagonal of the Fisher information matrix. In [10] the authors learned a metric with a neural network over the partial gradients w.r.t. the parameters, while in [11] the Neural Tangent Kernel based on the gradients is used to study the training of the network in the function space. It is not only the partial gradient w.r.t. parameters that carry important properties. For example in [12] the authors experimented with the norm of the input-output sensitivity, the Frobenius norm of the Jacobian matrix of the output w.r.t. input in case of simple architectures. Recent results show that under simple assumptions the maximal "capacity" (representational power) of deep ReLU networks is related to the arrangement of the polytopes in the input space [1] and to the properties of transition between linear regions $[13,3]$ instead of the exact number of polytopes with non-zero volume. Our last ingredient is Balcan and Blum's theory of similarity functions [8], which we will use as our foundation to show that we can define similarity functions in the gradient space that are at least as good similarity functions as the output of the network.

\subsection{Notations}

Let $f: \mathbb{R}^{d} \times \mathbb{R}^{|\theta|} \rightarrow \mathbb{R}$ be a feed-forward ReLU network, as a function of both the input data and the weights, containing $L$ hidden layers, $N$ neurons trained for solving a classification problem $P$ for some data distribution $D$. The activation function is ReLU: $h(x)=\max (0, x), x \in \mathbb{R}$. Let $N^{k}$ denote the number of neurons in the $k$-th layer and let $h_{\theta}^{k}(x) \in \mathbb{R}^{N^{k}}$ denote the output of the $k$-th layer for a given input vector $x$ and a fixed set of weights $\theta$. Denoting the weight vector after the last hidden layer, the discriminative layer, by $\theta_{L}$ we have $f_{\theta}(x)=\theta_{L}^{T} h_{\theta}^{L}(x)$. We denote the set of parameters as $\theta$ and the parameters of the $k$-th layer with $\theta_{k}$. The actual output of our model is $\operatorname{sgn}\left(f_{\theta}(x)\right)=$ $\operatorname{sgn}\left(\theta_{L}^{T} h_{\theta}(x)\right)$, i.e the network can be interpreted as a linear separator acting on $\operatorname{Im}\left(h_{\theta}\right) \subseteq \mathbb{R}^{N^{L}}$, the image of the nonlinear mapping $h_{\theta}: \mathbb{R}^{d} \rightarrow \mathbb{R}^{N^{L}}$. Following [1], we define an activation pattern with assigning a sign to each neuron in the network, $A=\left\{a_{l} ; l=1, . ., N\right\} \in\{-1,1\}^{N}$. For a particular input we refer $A(x ; \theta)=\left\{\operatorname{sgn}\left(\left[h_{\theta}(x)\right]_{l} ; l=1, . ., N\right\}\right.$ as the activation pattern assigned to an 
input $x$. An activation region with the corresponding fixed $\theta$ and $A$ is defined as $R(A ; \theta):=\left\{x \in \mathbb{R}^{d} \mid \operatorname{sgn}\left(\left[h_{\theta}(x)\right]_{l}\right)=a_{l}\right\}$, the set of input assigned to the same activation pattern. In comparison, linear regions are the input regions where the function defines different linear regions. We define tangent vectors as the change in the output with a directional derivative of $f_{\theta}(x)$ in the direction of $\left.g_{\theta}\left(x_{i}\right)=\left.\frac{\partial f_{\theta}(x)}{\partial \theta}\right|_{x=x_{i}}:\left(D_{g_{\theta}\left(x_{i}\right)} f\right)(\theta)=\frac{d}{d t}\left[f_{\theta}(x)+t g_{\theta}\left(x_{i}\right)\right)\right]\left.\right|_{t=0}$. We will refer $\nabla: \mathbb{R}^{d} \rightarrow \mathbb{R}^{|\theta|}$ as the tangent mapping of input at $\theta: \nabla_{\theta} f_{\theta}(x):=\frac{\partial f_{\theta}(x)}{\partial \theta}$. We denote the partial gradient vectors with $g_{\theta}(x) \in \mathbb{R}^{|\theta|}$. We denote the label of $x$ by $l(x)$, e.g. in case of binary classification $l(x) \in\{0,1\}$.

\section{Similarity functions}

First, we state the following definition from [8] which will be the measure of our similarity functions:

2.1. Definition. $A K(x, y)$ is a weakly $\gamma$-good similarity function for a learning problem $P$ if:

$$
E_{x, y \sim P}[K(x, y) \mid l(x)=l(y)] \geq E_{x, y \sim P}[K(x, y) \mid l(x) \neq l(y)]+\gamma .
$$

Note, the strong version of good similarity function says that a $K(x, y)$ is a good similarity function if $1-\epsilon$ probability mass of the examples are on average more similar to examples of the same category than to examples of another category. We consider the weakly good similarity because in this case we may investigate how the gap changes in case of a finite sample. We refer to $\gamma$ as the similarity gap.

We define the similarity function of the discriminative layer as $K_{f_{\theta}^{L}}(x, y)=$ $h_{\theta}^{L}(x)^{T} h_{\theta}^{L}(y)$ and the similarity function of the network as $K_{f_{\theta}}(x, y)=f_{\theta}(x) f_{\theta}(y)$. Now, let us consider the inner product in case of the tangent mapping. Before we argue about the metric in the tangent space we take a closer look on the structure of the Hessian in case of feed-forward ReLU networks. By definition the Hessian $H_{\theta}$ of the function induced by the network for a single output is a $|\theta| \times|\theta|$ sized matrix with $\left[H_{\theta}(x)\right]_{i j}=\frac{\partial^{2}\left[f_{\theta}(x)\right]}{\partial \theta_{i} \partial \theta_{j}}, \forall i, j \in\{1, . .,|\theta|\}$ which in our case is equal to the sum over the paths from the input to the output node where both $\theta_{i}$ and $\theta_{j}$ are present therefore if we split the Hessian into sub-blocks, where only elements from the same layer are present, these sub-blocks will be diagonal. This property suggest us to seek for a metric where the parameters of a layer in these sub-blocks may collide if necessary. Therefore we suggest a simple metric $M_{\theta} \in \mathbb{R}^{|\theta|^{2}}$ with $\left[M_{\theta}\right]_{i j}=\delta_{l_{f}(i), l_{f}(j)} \theta_{i} \theta_{j}$ where $\delta_{i, j}$ is one if $i=j$ and zero otherwise and $l_{f}(i)$ is the index of the layer of the $i$-th parameter. We define our new similarity function, the block diagonal similarity as

$$
K_{M_{\theta}}(x, y)=g_{\theta}(x)^{T} M_{\theta} g_{\theta}(y)=\sum_{i, j \in \theta \times \theta} \delta_{l_{f}(i), l_{f}(j)} \theta_{i} \theta_{j}\left[g_{\theta}(x)\right]_{i}\left[g_{\theta}(y)\right]_{j}
$$


where we utilized that the metric has a Cholesky decomposition as it is positive semi-definite. One of the most important properties of the block-diagonal similarity function is that in case of a single output the similarity function is equal, up to a constant, to the network output as

$$
\begin{aligned}
& \sum_{i, j \in \theta \times \theta} \delta_{l_{f}(i), l_{f}(j)} \theta_{i} \theta_{j}\left[g_{\theta}(x)\right]_{i}\left[g_{\theta}(y)\right]_{j}=\sum_{i, j \in \theta \times \theta} \delta_{l_{f}(i), l_{f}(j)} f_{\theta}(x) f_{\theta}(y) \\
& =\sum_{k=1}^{L} K_{f_{\theta}(x, y)} \approx O\left(K_{f_{\theta}}(x, y)\right) .
\end{aligned}
$$

Since $K_{f_{\theta}}(x, y)=\sum_{i \in \theta^{L}} \theta_{i}^{2}\left[h_{\theta}(x)\right]_{i}\left[h_{\theta}(y)\right]_{i}$ the following holds for the blockdiagonal similarity:

$$
\omega_{L} O\left(K_{f_{\theta}^{L}}(x, y)\right) \leq K_{M_{\theta}}(x, y) \leq \omega_{L}^{*} O\left(K_{f_{\theta}^{L}}(x, y)\right)
$$

where $\omega_{L}=\min _{i \in \theta^{L}} \theta_{i}^{2}$ and $\omega_{L}^{*}=\max _{i \in \theta^{L}} \theta_{i}^{2}$. Note, the similarity values are not necessarily positive.

Now, we decompose the gap and suggest a modified metric to increase the gap of the block-diagonal similarity function. As the norm of the gradient vectors highly affects the gap, we normalize to avoid linear increases in the gap. First, for simplicity we estimate the expectation $\mathbf{E}_{x, y \sim P}\left[K_{M_{\theta}}\left(x, x^{\prime}\right) \mid l(x)=l(y)\right]$ for a single layer and for a single label therefore the expectation is

$$
\begin{aligned}
& \sum_{i=1}^{|\theta|} \sum_{j=1}^{|\theta|} \mathbf{E}_{x, x^{\prime} \sim P}\left[\frac{\left[g_{\theta}(x)\right]_{i} \theta_{i} \theta_{j}\left[g_{\theta}\left(x^{\prime}\right)\right]_{j}}{\left\|M_{\theta}^{1 / 2} g_{\theta}(x)\right\|\left\|M_{\theta}^{1 / 2} g_{\theta}\left(x^{\prime}\right)\right\|}\right] \\
& \approx \frac{1}{\left|T^{(+)}\right|^{2}} \sum_{t_{1}, t_{2} \in T^{(+)} \times T^{(+)}} \frac{1}{\left\|\hat{g}_{\theta}\left(x_{t_{1}}\right)\right\|\left\|\hat{g}_{\theta}\left(x_{t_{1}}\right)\right\|} \sum_{i=1}^{|\theta|} \sum_{j=1}^{|\theta|}\left[g_{\theta}\left(x_{t_{1}}\right)\right]_{i} \theta_{i} \theta_{j}\left[g_{\theta}\left(x_{t_{2}}\right)\right]_{j} \\
& =\sum_{i, j \in \theta \times \theta} \frac{1}{\left|T^{(+)}\right|^{2}} \sum_{t_{1}, t_{2} \in T^{(+)} \times T^{(+)}} \frac{\left[g_{\theta}\left(x_{t_{1}}\right)\right]_{i} \theta_{i} \theta_{j}\left[g_{\theta}\left(x_{t_{2}}\right)\right]_{j}}{\left\|\hat{g}_{\theta}\left(x_{t_{1}}\right)\right\|\left\|\hat{g}_{\theta}\left(x_{t_{1}}\right)\right\|}=\sum_{i, j \in \theta \times \theta}\left[\hat{\psi}_{M_{\theta}}^{+}\right]_{i j}
\end{aligned}
$$

where we denote the set of examples with label "+" as $T^{(+)}$, a subset of known examples $T$ and $M_{\theta}^{1 / 2} g_{\theta}(x)$ with $\hat{g_{\theta}}(x)$. Similar calculation can be applied if the labels are different thus

$$
\begin{aligned}
\gamma_{M_{\theta}} & =\mathbf{E}_{x, y \sim P}\left[K_{M_{\theta}}\left(x, x^{\prime}\right) \mid l(x)=l(y)\right]-\mathbf{E}_{x, y \sim P}\left[K_{M_{\theta}}\left(x, x^{\prime}\right) \mid l(x) \neq l(y)\right] \\
& \approx \sum_{i, j \in \theta \times \theta}\left[\hat{\psi}_{M_{\theta}}^{(+)}\right]_{i j}-\left[\hat{\psi}_{M_{\theta}}^{(-)}\right]_{i j}=\sum_{i, j \in \theta \times \theta}\left[\hat{\psi}_{M_{\theta}}\right]_{i j} .
\end{aligned}
$$

Therefore if we consider multiple layers, where $\left[M_{\theta}\right]_{i j}=0$ if $l_{f}(i) \neq l_{f}(j)$, the similarity gap can be approximated as

$$
\gamma_{M_{\theta}} \approx \sum_{i, j \in \theta \times \theta} \delta_{l_{f}(i), l_{f}(j)}\left[\hat{\psi}_{M_{\theta}}\right]_{i j}=\sum_{k \in 1, \ldots, L} \sum_{i, j \in \theta^{k} \times \theta^{k}}\left[\hat{\psi}_{M_{\theta}}\right]_{i j} .
$$


Note, with $|T| \rightarrow \infty$ the error of our approximation converges to zero with probability one, moreover this convergence is true for every element in $\hat{\psi}_{M_{\theta}}$. An additional consequence that we can partition the elements of the similarity gap into disjoint sets and examine each independently. Observe, the elements of $\hat{\psi} \in \mathbb{R}$ therefore let us define for every pair their "importance" in the gap as $i m p_{i, j}=\max \left\{0,\left[\hat{\psi}_{M_{\theta}}\right]_{i j}\right\}$. By setting the elements in the metric with low or negative importance we can define a sparse block-diagonal similarity with an additional step, normalization. However, our new metric can have the same dimensions as the original block-diagonal. Thus we can argue that according to $i m p_{i}=\sum_{j} i m p_{i j}, \forall i \in \theta$ we can select the most important parameters and delete rows and columns associated with less important parameters and form the elementwise block-diagonal metric.

So far we assumed arbitrary input and do not take advantage of the gradient graph of the network. To show a case when the normalized sparse block-diagonal similarity has better similarity gap than the original normalized block-diagonal similarity we will assume that our input is a subgaussian random vector [14] with zero mean and variance one, e.g. the input is element-wise standard normalized. Additionally, observe that in ReLU networks the partial gradients can be expressed as $g_{\theta}(x)=S_{\theta}(x) x$ where $S_{\theta}(x)_{i, j}=\left.\frac{\partial^{2} f_{\theta}(x)}{\partial \theta \partial x}\right|_{\theta, x}$ a $d \times|\theta|$ sized matrix and inside an activation region $S_{\theta}(x)=S_{\theta}(A(x))$ is identical for each $x \in A$ thus $g_{\theta}(x)=S_{\theta}(A(x)) x$. Due the complexity of the proof and the page limit we only mention that, following Theorem 2.1 in [15], the norm of the elementwise sparse vector for points in the activation region $A$ is concentrated as for all $\delta>0$

$$
P\left(\left\|S_{\theta}^{*}(A(x)) x\right\|_{2}^{2}>\operatorname{Tr}\left[S_{\theta}^{*}(A(x)) S_{\theta}^{*}(A(x))^{T}\right](1+4 \delta) ; x \in A\right) \leq e^{-\delta}
$$

where $S_{\theta}^{*}(A(x))$ is the same as $S_{\theta}(A(x))$ without the removed rows and columns and therefore the similarity gap in case of the normalized elementwise sparse block-diagonal similarity is related to the ratio $\frac{\operatorname{Tr}\left[S_{\theta}(A(x)) S_{\theta}(A(x))^{T}\right]}{\operatorname{Tr}\left[S_{\theta}^{*}(A(x)) S_{\theta}^{*}(A(x))^{T}\right]}$.

We experimented on the CIFAR-10 dataset [16] with a simple feed-forward network with five layers ${ }^{1}$. We ranked the parameters per layer according the elementwise block-diagonal metric and deleted the parameters with low "importance". Results in Fig. 1 indicate that the gap can be increased but further, more detailed experimentation needed to understand how the gap is actually increasing.

\section{Conclusions}

In this paper we defined similarity functions over ReLU networks based on the gradient structure and investigated the similarity gap. Furthermore, we introduced a measure to rank the parameter pairs in the network according to their importance in the similarity gap. In the future we plan to extend our work to other network structures as our findings were limited to feed-forward fully connected ReLU networks.

\footnotetext{
${ }^{1}$ Experiments are available at https://github.com/danielracz/gradsim
} 
ESANN 2021 proceedings, European Symposium on Artificial Neural Networks, Computational Intelligence and Machine Learning. Online event, 6-8 October 2021, i6doc.com publ., ISBN 978287587082-7.

Available from http://www.i6doc.com/en/.

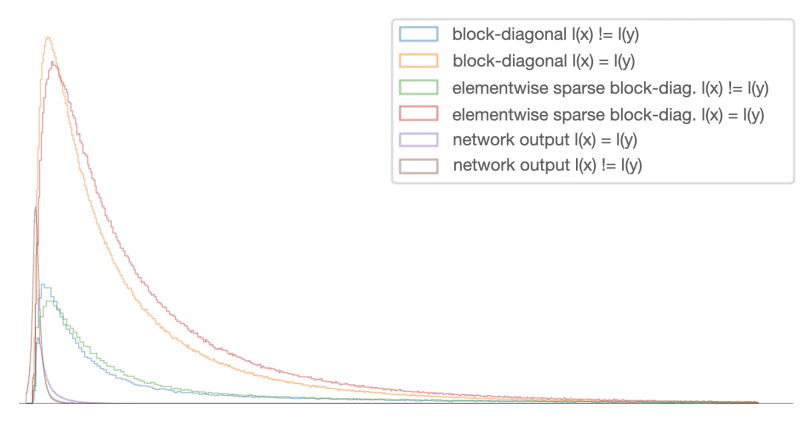

Fig. 1: Distribution of the network output, the elementwise sparse block-diagonal and the block-diagonal similarity values.

\section{References}

[1] Boris Hanin and David Rolnick. Complexity of linear regions in deep networks. In International Conference on Machine Learning, pages 2596-2604, 2019.

[2] Xiao Zhang and Dongrui Wu. Empirical studies on the properties of linear regions in deep neural networks. arXiv preprint arXiv:2001.01072, 2020.

[3] Boris Hanin, Ryan Jeong, and David Rolnick. Deep relu networks preserve expected length. arXiv preprint arXiv:2102.10492, 2021.

[4] James Martens and Roger Grosse. Optimizing neural networks with kronecker-factored approximate curvature. In International conference on machine learning, pages 2408$2417,2015$.

[5] Shun-ichi Amari. Neural learning in structured parameter spaces-natural Riemannian gradient. In NIPS, pages 127-133. Citeseer, 1996.

[6] Yann Ollivier. Riemannian metrics for neural networks i: feedforward networks. Information and Inference: A Journal of the IMA, 4(2):108-153, 2015.

[7] N. N. C̆encov. Statistical decision rules and optimal inference. American Mathematical Society, 53, 1982.

[8] Maria-Florina Balcan, Avrim Blum, and Nathan Srebro. A theory of learning with similarity functions. Machine Learning, 72(1-2):89-112, 2008.

[9] Tommi S Jaakkola, David Haussler, et al. Exploiting generative models in discriminative classifiers. Advances in neural information processing systems, pages 487-493, 1999.

[10] Balint Daroczy, Rita Aleksziev, and András Benczúr. Tangent space separability in feedforward neural networks. In proc. of BFO at NeurIPS 2019.

[11] Arthur Jacot, Franck Gabriel, and Clément Hongler. Neural tangent kernel: Convergence and generalization in neural networks. NeurIPS 2018.

[12] Roman Novak, Yasaman Bahri, Daniel A Abolafia, Jeffrey Pennington, and Jascha SohlDickstein. Sensitivity and generalization in neural networks: an empirical study. arXiv preprint arXiv:1802.08760, 2018.

[13] Maithra Raghu, Ben Poole, Jon Kleinberg, Surya Ganguli, and Jascha Sohl Dickstein. On the expressive power of deep neural networks. In Proc. of ICML'17.

[14] Tor Lattimore and Csaba Szepesvári. Bandit algorithms. Cambridge University Press, 2020.

[15] Daniel Hsu, Sham Kakade, Tong Zhang, et al. A tail inequality for quadratic forms of subgaussian random vectors. Electronic Communications in Probability, 17, 2012.

[16] Alex Krizhevsky, Geoffrey Hinton, et al. Learning multiple layers of features from tiny images. Technical Report TR-2009, University of Toronto, Toronto, 2009. 